\title{
Time scale dependent sensitivities of the XinAnJiang model parameters
}

\author{
Minjiao $\mathrm{Lu}^{1,2}$ and $\mathrm{Xiao} \mathbf{L i}^{1}$ \\ ${ }^{1}$ Nagaoka University of Technology, Niigata, Japan \\ ${ }^{2}$ Chongqing Jiaotong University, Chongqing, China
}

\begin{abstract}
:
The objective of this study was to investigate the sensitivities of the parameters of the XinAnJiang model, hereinafter referred to as XAJ model. The XAJ model is the most popular rainfall-runoff model in China, and widely used all over the world. There were fifteen parameters in the modified XAJ model used in this study. Understanding the sensitivities is undoubtedly crucial for parameter optimization, even for manual calibration by trial and error. A sensitivity analysis technique proposed by Morris was used to analyze parameter sensitivities at time scales of year, month and day. The sensitivities of these parameters were shown to change. At annual scale, the parameters for input data adjustment are most sensitive. On the other hand, the parameters concerning runoff component separation and runoff concentration are sensitive at daily scale. The parameters relating to runoff generation are less sensitive at all three temporal scales. Additionally, strong interactions between the parameters were detected at all three temporal scales. The time scale dependent nature of the sensitivities offers the possibility to design more efficient optimization schemes for automatic model calibration of the XAJ model.
\end{abstract}

KEYWORDS sensitivity analysis; the XinAnJiang model; temporal scale; parameter interaction

\section{INTRODUCTION}

Recently, hydrological models ranging from conceptual models to physical models have been becoming more and more comprehensive and complicated. Many modern models have more than ten parameters that may be sensitive, conditionally sensitive or insensitive. Considering their physical meanings and roles in the model, their sensitivity should differ at different time scales. For example, the parameter controlling the runoff concentration of surface water will be very sensitive at short time scales, but less sensitive or completely insensitive at time scales much longer than the concentration time of the catchment. The high dimensionality and insensitivities of model parameters, together with their interaction, make automatic or manual model calibration very difficult. It is essential for a modeler to have a better understanding of parameter sensitivity in all phases of hydrological modeling: formulation, calibration, evaluation, and application of a new model. For example, understanding parameter sensitivity can be helpful to avoid over-parameterization in model formulation, a frequently addressed problem for hydrological models, especially distributed ones (Beven, 1989).
Generally, sensitivity is a measure of the effect of change in one factor on another factor. In hydrological modeling, it can be the effect of changes in parameters on model outputs, which may be a measure of goodness of fit such as error variance, root mean square error or the modeling efficiency of Nash and Sutcliffe (1970). Parametric sensitivity is a vital issue of most optimization techniques using such measures as an objective function. However, other facets of sensitivity need to be recognized. The identification of insensitive or conditionally insensitive parameters will be of great importance in the formulation of hydrological models.

Vemuri et al. (1969) pointed out that sensitivity analysis should be an integral part of nearly every hydrological study. McCuen (1973) showed the importance of sensitivity analysis and proposed a mathematical framework of sensitivity. Bathurst (1986) made a sensitivity analysis of the Système Hydrologique Européen based on simulations of two streamflow hydrographs from an upland catchment in mid-Wales. Lenhart et al. (2002) carried out a comparison of two simple approaches of sensitivity analysis using the physically based, continuous time hydrological model SWAT (Soil and Water Assessment Tool), demonstrating the sensitivities of the parameters. There are numerous papers reporting results of sensitivity analysis of different methods and models. However, most of them, including the recent ones, are local approaches: only local derivatives of the objective function computed directly or indirectly are considered. The major drawback of these approaches is their inability to account for parameter interactions, and then their proneness to underestimating true model sensitivities.

On the other hand, global sensitivity analysis techniques have received considerable attention in recent years. Many techniques have been developed that can be applied even to non-linear, non-monotonic models (Saltelli et al., 2004). Tang et al. (2007) compared four sensitivity analysis methods, one local and three global, and showed the superiority of global methods.

The purpose of this study is to investigate the sensitivities of the parameters in the XinAnJiang model (Zhao, 1992), hereinafter referred to as XAJ model, and their dependency on temporal scales. There are fifteen parameters in the modified XAJ model used in this study. A global sensitivity analysis technique proposed by Morris (1991) was used to analyze their sensitivity at time scales of year, month and day. It is shown that the sensitivities of these parameters change according to the time scales. The time scale dependency of the sensitivities will provide important information in hydrological modeling practices. 


\section{THE XAJ MODEL}

The XAJ model is a conceptual hydrological model to simulate runoff generation and concentration within a catchment. It is widely used in humid and semi-arid regions of China. There are fifteen parameters in the modified XAJ model used in this study. This model includes five parts: input data adjustment, runoff generation and evapotranspiration, runoff component separation, runoff routing, and channel routing. The model parts, except the channel routing are described briefly in the following subsections. Channel routing is not considered in this study as the catchment is not divided into sub-catchments.

\section{Input data adjustment}

The XAJ model takes rainfall and potential evaporation as inputs. Though there are many problems with this approach, the potential evaporation, $E_{p}$, is calculated from pan evaporation as follows,

$$
E_{p}=C_{e p} E_{\text {pan }}
$$

where $E_{\text {pan }}$ is pan evaporation routinely observed at many hydrological stations, and $C_{e p}$ is an adjustment coefficient for potential evaporation.

In the original XAJ model, the catchment is usually divided into Thiessen polygons. The runoff from each polygon is calculated from corresponding rainfall data independently and routed to the basin outlet using the Muskingum method. Adjustment of rainfall is not considered.

However, the model was used in a pure lumped way in this study. The areal average rainfall was inputted to the model representing the whole catchment. In order to take into account the possible errors caused by gauge losses, altitudinal distribution of rainfall, and the representativeness of the gauge network, an adjustment coefficient, $C_{p}$, was introduced to derive adjusted rainfall, $P$, from areal average rainfall, $P_{a}$, as follows.

$$
P=C_{p} P_{a}
$$

\section{Runoff generation and evapotranspiration}

The XAJ model is a so-called probability distributed model (Moore, 1985, 2007). A pure statistical distribution of tension water capacity of all points, WPM, over the catchment is assumed to be represented by a single parabolic curve defined as

$$
\frac{f}{F}=1-(1-i m p)\left\{1-\frac{W P M}{W M M}\right\}^{b}
$$

where imp is the portion of impervious area; $W M M$ is maximum value of $W P M$ within a catchment; $b$ is a shape parameter; and $\frac{f}{F}$ is the portion of catchment area having tension water capacity smaller than WPM. Note the impervious area is included in $\frac{f}{F}$. When the tension water capacity smaller than $a$ is filled as shown in the left panel of Figure 1, the catchment mean tension water storage, $W$, can be derived by

where

$$
\begin{aligned}
W & =\int_{0}^{a}\left(1-\frac{f}{F}\right) d W P M \\
& =W M\left\{1-\left(1-\frac{a}{W M M}\right)^{1+b}\right\}
\end{aligned}
$$

$$
W M=(1-i m p) \frac{W M M}{1+b}
$$

is the catchment mean tension water capacity.

In the XAJ model, only saturation excess runoff is simulated. Runoff occurs only over the points where the tension water capacities are filled. The runoff caused by rainfall, $P$, falling over a catchment with initial storage, $W$, will be

$$
R=\int_{a}^{a+P} \frac{f}{F} d W P M
$$

The relationship between rainfall and runoff is shown in the right panel of Figure 1. For all initial storages, the line slope becomes steeper when rainfall increases and finally reaches 45 degree. This means that the runoff ratio equal to the saturated area, $\frac{f}{F}$ increases and finally reaches unity when
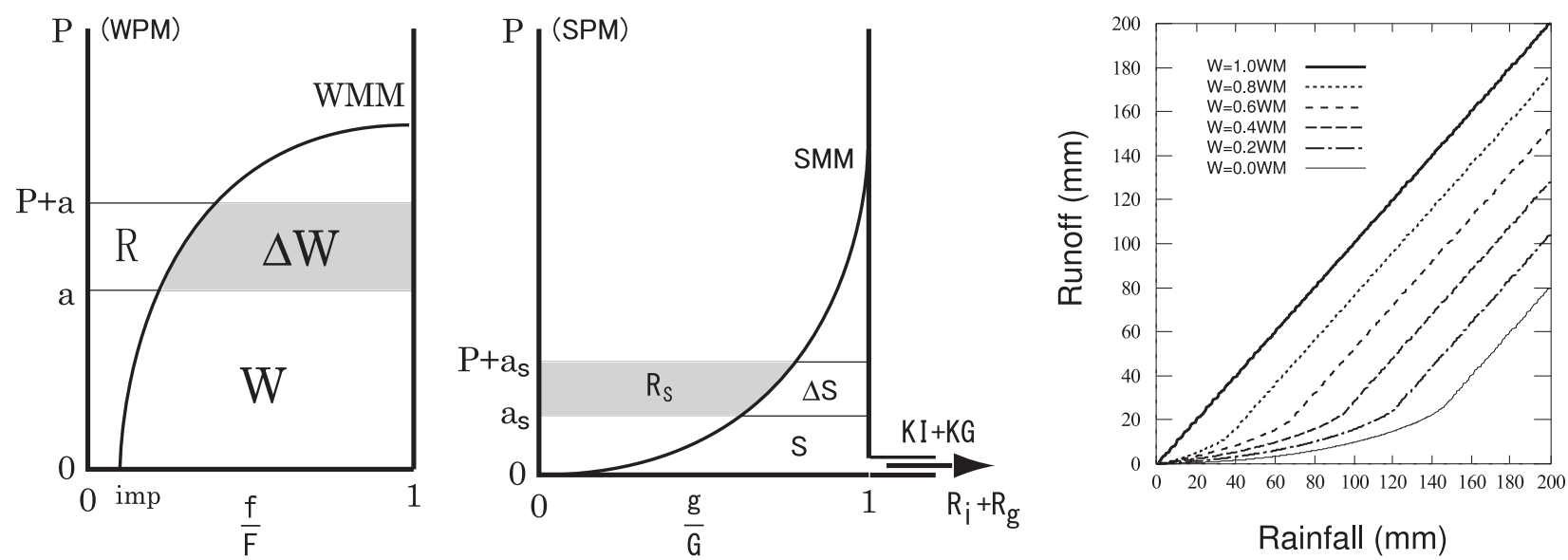

Figure 1. Schematics of the XAJ model (left: distribution of tension water over the catchment; center: distribution of free water; and right: rainfall-runoff relationship based on the tension water distribution) 
rainfall continues.

The increment of the catchment mean tension water storage, $\Delta W$, will be

$$
\Delta W=\int_{a}^{a+P}\left(1-\frac{f}{F}\right) d W P M
$$

Obviously, $\Delta W=P-R$.

In this model, a three layer evapotranspiration scheme is used. The soil is divided into three layers, namely up layer, low layer and deep layer. $W M$ is also divided into $W U M, W L M$ and $W D M . \Delta W$ is first used to fill $W U M$, then $W L M$, and finally $W D M$. The soil water is evapotranspired from up layer, low layer and finally, the deep layer. The evapotranspiration rate is calculated by

$$
E T= \begin{cases}E_{p} & W U>0 \\ E_{p} \max \left(\frac{W L}{W L M}, C\right) & W U=0 \text { and } W L>0 \\ E_{p} C & W U=0 \text { and } W L=0\end{cases}
$$

where $C$ is a parameter controlling the evapotranspiration under dry condition. After calculation of evapotranspiration, the storage $W$ will be updated as $W=W U+W L+W D$, to be used as initial storage in the next step.

For runoff generation and evapotranspiration, six parameters, $W U M, W L M, W D M, b, i m p$ and $C$ are used.

\section{Separation of runoff components}

The runoff calculated by Equation (6) will be separated into two or three runoff components. The XAJ model with three runoff components, surface runoff, interflow and baseflow is used in this study. As mentioned, runoff will occur only at the saturated portion of the catchment, $\left(\frac{f}{F}\right)_{R}$. Another single parabolic curve shown in the center panel of Figure 1

$$
\frac{g}{G}=1-\left\{1-\frac{S P M}{S M M}\right\}^{E X}
$$

is introduced to represent the statistical distribution of the free water capacities at all points within this area. SPM, $S M M, E X$ and $\frac{g}{G}$ are variables for free water corresponding to $W P M, W M M, b$ and $\frac{f}{F}$ of tension water. It is worth noting that the impervious area is not included in Equation (9) and $\frac{g}{G}$ is the portion relative to $\left(\frac{f}{F}\right)_{R}-i m p$. In a similar way, when the free water capacity smaller than $a_{s}$ is filled, the areal mean free water storage, $S$ can be derived by

$$
\begin{aligned}
S & =\int_{0}^{a_{S}}\left(1-\frac{g}{G}\right) d S P M \\
& =S M\left\{1-\left(1-\frac{a_{S}}{S M M}\right)^{1+E X}\right\}
\end{aligned}
$$

where

$$
S M=\frac{S M M}{1+E X}
$$

is the areal mean free water capacity.

Using this distribution, surface runoff can be derived in the same way as total runoff in Equation (6).

$$
R_{S}=i m p \cdot P+\left\{\left(\frac{f}{F}\right)_{R}-i m p\right\} \int_{a_{s}}^{a_{s}+P} \frac{g}{G} d S P M
$$

And interflow and baseflow are computed by

$$
\begin{aligned}
& R_{i}=S \cdot K I \cdot\left\{\left(\frac{f}{F}\right)_{R}-i m p\right\} \\
& R_{g}=S \cdot K G \cdot\left\{\left(\frac{f}{F}\right)_{R}-i m p\right\}
\end{aligned}
$$

where $K I$ and $K G$ are parameters controlling free water drainage and the ratio of interflow to baseflow. The new free water storage, $S$, can be derived by adding increment caused by rainfall and subtracting drainages. Because $\left(\frac{f}{F}\right)_{R}$ changes with every time step, $S$ always needs to be converted to the depth over the new saturated area.

For separation of runoff components, four parameters, $S M, E X, K I$ and $K G$, are used. Usually, $0<K I+K G<1$.

\section{Runoff routing}

The three runoff components are then routed using three linear reservoirs as follows:

$$
\begin{aligned}
& Q_{s}(t+1)=c_{s} Q_{s}(t)+\left(1-c_{s}\right) R_{s} \\
& Q_{i}(t+1)=c_{i} Q_{i}(t)+\left(1-c_{i}\right) R_{i} \\
& Q_{g}(t+1)=c_{g} Q_{g}(t)+\left(1-c_{g}\right) R_{g}
\end{aligned}
$$

where $c_{s}, c_{i}$ and $c_{g}$ are three recession coefficients. Usually, $0<c_{S}<c_{i}<c_{g}<1$.

\section{SENSITIVITY ANALYSIS}

A global sensitivity analysis method proposed by Morris (1991) was used in this study. Probably, this method has been used most widely due to the ease of interpreting the results. The guiding philosophy of this method is to determine which factor may be considered to have effects which are (a) negligible, (b) linear and additive, or (c) nonlinear or involved in interactions with other factors.

For simplicity, consider a set of factors as a vector in a $k$-dimensional unit hypercube and each factor may take one of $p$ values uniformly discretized from 0 to 1 , namely 0,1 / $(p-1), \ldots,(p-2) /(p-1), 1$. The value space of the factors, $\Omega$, will then be a $k$-dimensional $p$-level grid. For a vector $\mathbf{x}$ in $\Omega$, an elementary effect of the $i$-th factor $d_{i}(\mathbf{x})$, named by Morris, is defined as

$$
d_{i}(\mathbf{x})=\frac{y\left(\mathbf{x}+\mathbf{e}_{i} \Delta\right)-y(\mathbf{x})}{\Delta}
$$

where $y$ is the model output, objective function value in this study; $\Delta$ is a predetermined multiple of $1 /(p-1)$; and $\mathbf{x}+$ $\mathbf{e}_{i} \Delta$ is the transformed vector still in $\Omega$, where $\mathbf{e}_{i}$ is a vector of zeros but with a unit as its $i$-th component. The finite distribution of elementary effects associated with the $i$-th factor, denoted as $\boldsymbol{F}_{i}$, can be obtained by randomly sampling different $\mathbf{x}$ in $\Omega$. There are $p^{k-1}[p-\Delta(p-1)]$ elements for each $\boldsymbol{F}_{i}$. Computing elementary effects of all elements is obviously impossible. Usually, the distribution's statistics are estimated by sampling a number, $r$, of elementary effects from $\boldsymbol{F}_{i}$. From these $r$ elementary effects, two sensitivity measures proposed by Morris can be calculated for each factor. Measure $\mu_{i}$ 
Table I. Transformation from sensitivity analysis variables to model parameters

\begin{tabular}{cclc}
\hline Opt. Var. & Range & \multicolumn{1}{c}{ Transformation } & Parameter range \\
\hline$x_{1}$ & $0.0-1.0$ & $C_{p}=0.8+0.4 x_{1}$ & $0.8-1.2$ \\
$x_{2}$ & $0.0-1.0$ & $C_{e p}=2 x_{2}$ & $0-2.0$ \\
$x_{3}$ & $0.0-1.0$ & $b=0.1+0.2 x_{3}$ & $0.1-0.3$ \\
$x_{4}$ & $0.0-1.0$ & $i m p=0.05 x_{4}$ & $0-0.05$ \\
$x_{5}$ & $0.0-1.0$ & $W U M=10+20 x_{5}$ & $10-30$ \\
$x_{6}$ & $0.0-1.0$ & $W L M=60+60 x_{6}$ & $60-120$ \\
$x_{7}$ & $0.0-1.0$ & $W D M=10+90 x_{7}$ & $10-100$ \\
$x_{8}$ & $0.0-1.0$ & $C=0.1+0.2 x_{8}$ & $0.1-0.3$ \\
$x_{9}$ & $0.0-1.0$ & $S M=1.0+49.0 x_{9}$ & $1.0-50.0$ \\
$x_{10}$ & $0.0-1.0$ & $E X=0.5+2.0 x_{10}$ & $0.5-2.5$ \\
$x_{11}$ & $0.0-1.0$ & $K I=0.001+0.95 x_{11}$ & $0.001-0.951$ \\
$x_{12}$ & $0.0-1.0$ & $K G=0.001+0.95(1-K I) x_{12}$ & $0.001-0.95(1-\mathrm{KI})$ \\
$x_{13}$ & $0.0-1.0$ & $c_{s}=0.01+0.99 c_{i} x_{13}$ & $0.01-c_{i}$ \\
$x_{14}$ & $0.0-1.0$ & $c_{i}=0.01+0.99 c_{g} x_{14}$ & $0.01-c_{g}$ \\
$x_{15}$ & $0.0-1.0$ & $c_{g}=0.97+0.025 x_{15}$ & $0.97-0.995$ \\
\hline
\end{tabular}

$$
\mu_{i}=\frac{1}{r} \sum_{j=1}^{r} d_{i}\left(\mathbf{x}^{(j)}\right)
$$

estimates the overall effect of the factor on the model output; and measure $\sigma_{i}$

$$
\sigma_{i}=\sqrt{\frac{1}{r} \sum_{j=1}^{r}\left(d_{i}\left(\mathbf{x}^{(j)}\right)-\mu_{i}\right)^{2}}
$$

estimates the ensemble of the second- and higher-order effects in which the factor is involved. Here $\mathbf{x}^{(j)}$ is the $\mathrm{j}$-th set of factors sampled from $k$-dimensional unit hypercube.

It is clear that $\mu$ is not a good measure for non-monotonic models because some elementary effects will cancel each other. Campolongo et al. (2007) proposed another measure $\mu^{*}$ as

$$
\mu_{i}^{*}=\frac{1}{r} \sum_{j=1}^{r}\left|d_{i}\left(\mathbf{x}^{(j)}\right)\right|
$$

In this study, $\mu^{*}$ and $\sigma$ were used. The larger the measure $\mu^{*}$, the more sensitive that factor. The larger the measure $\sigma$, the stronger the effects from other factors that factor accepts. A large $\sigma_{i}$ implies that other factors also have significant impacts on $d_{i}$ and make them differ from its mean value $\mu_{i}$.

In order to calculate $\mu^{*}$ and $\sigma$ of all factors, $2 \times k \times r$ model evaluations are necessary if $\mathbf{x}$ is randomly selected. The experimental plan proposed by Morris (1991) makes it possible by only $(k+1) \times r$ model evaluations. It is composed of an individually randomized 'one-factor-at-a-time' approach, referred to as OAT. In each model evaluation, only one factor is changed so that the change in the output can be unambiguously attributed to the changed factor. The impact of changing one factor at a time is evaluated in turn. For the implementation details of the Morris method, refer to Saltelli et al. (2004).

In the sensitivity analysis of XAJ model parameters, $k$ $=15$ is the dimension of the parameter space and $r$ was set to 4 , which is the smallest number showing similar results with larger $r$ values. $(k+1) \times r$ sets of the factors were generated using OAT. Then they were transformed into $(k$ $+1) \times r$ sets of XAJ model parameters using the equations listed in Table I. This transformation reflects the range of the parameters obtained in vast model applications in China, and also keeps all constraints, such as $0<K I+K G<1$ and $0<c_{s} \leq c_{i} \leq c_{g}<1$, valid. For each parameter set, a simulation run was carried out to obtain a time series of discharge from time series of rainfall and potential evaporation. Then the objective function, modeling efficiency of Nash and Sutcliffe (1970), NSE, is calculated using observed and simulated discharge data, $Q_{o b s}$ and $Q_{\text {sim }}$.

$$
\mathrm{NSE}=1-\frac{\sum_{t=1}^{n}\left(Q_{o b s}(t)-Q_{s i m}(t)\right)^{2}}{\sum_{t=1}^{n}\left(Q_{o b s}(t)-\overline{Q_{o b s}}\right)^{2}}
$$

where $t$ and $n$ are time and the number of data points.

In this study, the daily rainfall and pan evaporation data of the Misai basin located in south east China, a basin with drainage area of $797 \mathrm{~km}^{2}$, was used to drive the XAJ model. Daily discharge data were used to evaluate the modeling efficiency of Nash and Sutcliffe (1970). The XAJ model was run at daily scale from 1982 to 1988 , and from the simulated daily discharge, the monthly and annual discharge data were calculated. Three modeling efficiencies of Nash and Sutcliffe (1970) were calculated from annual, monthly and daily discharge data, respectively. They were used in the evaluation of parameter sensitivities. In order to avoid the effects from initial conditions, the data in the first year were not used in the calculation of the modeling efficiency of Nash and Sutcliffe (1970).

\section{RESULTS AND CONCLUSIONS}

The left panel of Figure 2 shows the sensitivity measures $\mu^{*}$ and $\sigma$ at annual scale. The variable names of the XAJ model parameters are displayed. Actually, they are sensitivity measures of the factors $\mathrm{x}$. In most parameter optimization practices in hydrological modeling, a transformation to consider constraints is necessary. In such cases, the sensitivities of $\mathrm{x}$ will be more direct and useful. Clearly, the parameters $x_{1}$ and $x_{2}$, and consequently $C_{p}$ and $C_{e p}$, are much more sensitive than all other factors. Also they display much larger values of the measure $\sigma$. This means that there is strong interaction between these two factors. Physically, 

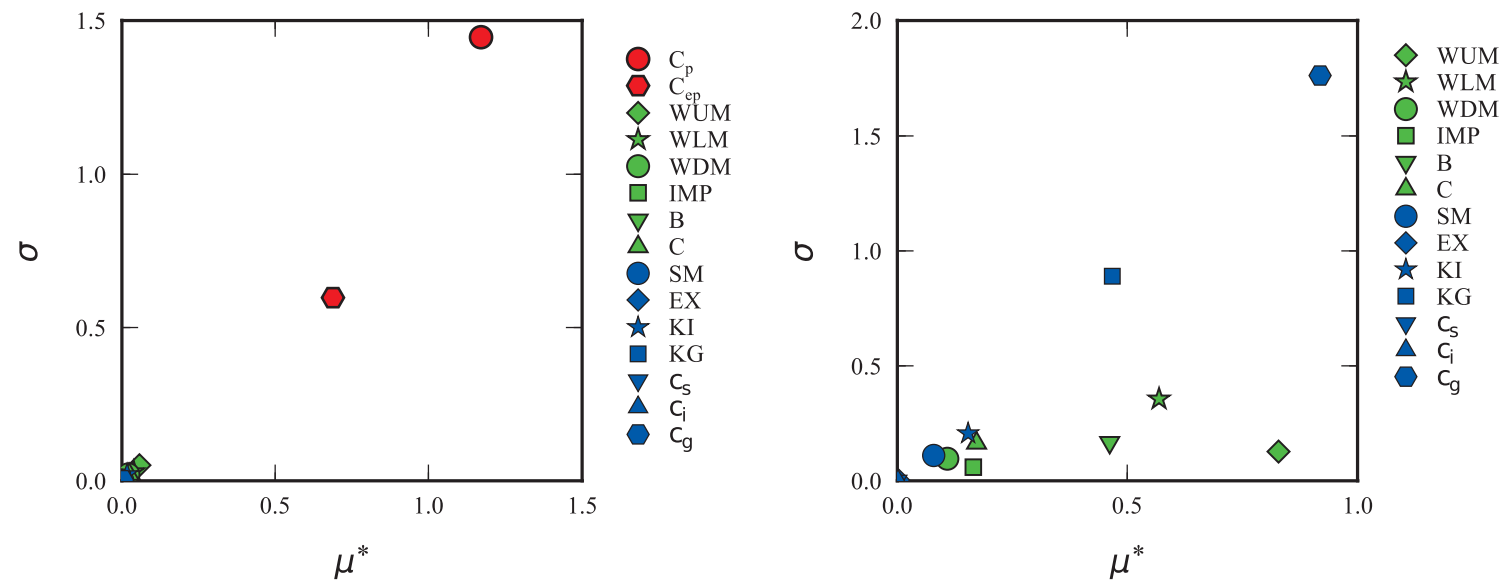

Figure 2. Sensitivity measures of XAJ model parameters at annual scale (left: annual scale of all 15 parameters; right: annual scale while keeping $C_{p}$ and $C e p$ constant)
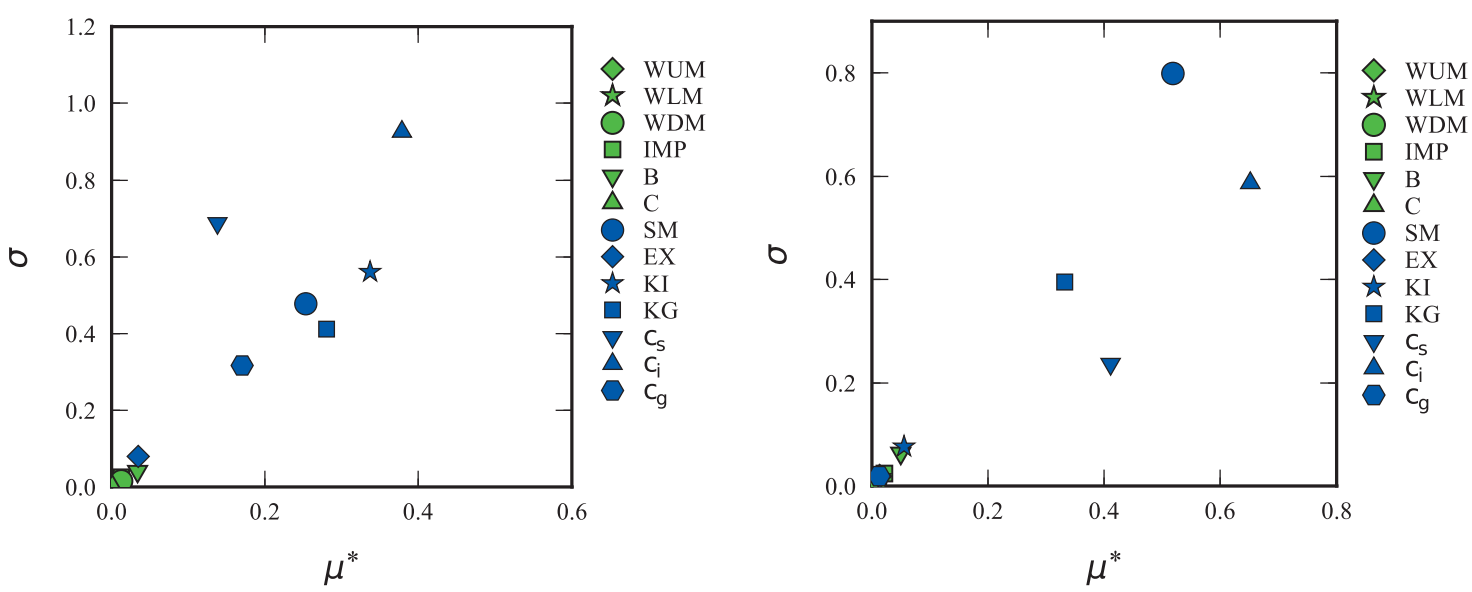

Figure 3. Sensitivity measures of XAJ model parameters at monthly and daily scale while keeping $C_{p}$ and $C_{e p}$ constant (left: monthly scale; right: daily scale)

increases in $C_{p}$ will increase annual discharge, and increases in $C_{e p}$ will decrease annual discharge. The effect caused by increasing $C_{p}$ will be partly canceled by increasing $C_{e p}$. The strong interaction shown here indicates this cancellation. This implies that it is possible to optimize $C_{p}$ and $C_{e p}$ only at annual scale with limited effects caused by other parameters.

Figure 3 shows the sensitivity measures $\mu^{*}$ and $\sigma$ at monthly and daily scales. In the monthly and daily sensitivity analysis, $C_{p}$ and $C_{e p}$ are set to constants so that the two most sensitive factors $x_{1}$ and $x_{2}$ will not affect other factors. This is done by changing $C_{p}=0.8+0.4 x_{1}$ and $C_{e p}=2 x_{2}$ in Table I to $C_{p}=1.1$ and $C_{e p}=0.85$. At both time scales, factors related to parameters controlling the runoff routing, namely $S M, E X, K I, K G, c_{s}, c_{i}$ and $c_{g}$ are relatively sensitive, though their relative sensitivities change. Among these parameters, $E X$ and $c_{g}$ seem to be insensitive, however, insensitivity of $c_{g}$ is partly caused by the narrow range set in Table I. This setting is based on the factor that $c_{g}$ can be determined from the recession curves quite accurately. It was also shown that factors related to parameters controlling runoff generation, namely $W U M, W L M, W D M, b$, imp and
C were insensitive at both time scales.

Interestingly, the factors controlling runoff generation become sensitive again at annual scale while keeping $C_{p}$ and $C_{e p}$ constant, as shown in the right panel of Figure 2. Besides $c_{g}$ and $K G$ which control recession of the computed hydrograph, $W U M, W L M$ and $B$ show relatively large $\mu^{*}$ values. Furthermore, their $\sigma$ values are also relatively small. These parameters seem to have relatively weak interactions with other parameters. Though not as significant as $C_{p}$ and $C_{e p}$, their effects on annual evaporation and discharge, consequently annual water balance can be deciphered.

From above results, it is possible to conclude:

1. At annual scale, the parameters controlling data adjustment, $C_{p}$ and $C_{e p}$, are most sensitive.

2. While keeping $C_{p}$ and $C_{e p}$ constant, parameters controlling runoff routing are sensitive at monthly and daily scale; parameters controlling runoff generation are insensitive at both time scales.

3. While keeping $C_{p}$ and $C_{e p}$ constant, parameters controlling runoff generation become sensitive again at annual scale together with several parameters controlling runoff routing. 
The time scale dependent nature of the sensitivities will make it possible to design more efficient optimization schemes for automatic model calibration of the XAJ model. For example, this allows the optimization of several parameters at the time scale at which they are sensitive, with limited impacts caused by other parameters. For the XAJ model, this implies the potential to optimize $C_{p}$ and $C_{e p}$ at annual scale while fixing other parameters to their commonly used values.

\section{REFERENCES}

Bathurst J. 1986. Sensitivity analysis of the Système Hydrologique Européen for an upland catchment. Journal of Hydrology 87: 103-123. DOI: 10.1016/0022-1694(86)90117-4.

Beven K. 1989. Changing ideas in hydrology - the case of physically-based models. Journal of Hydrology 105: 157172. DOI: 10.1016/0022-1694(89)90101-7.

Campolongo F, Cariboni J, Saltelli A. 2007. An effective screening design for sensitivity analysis of large models. Environmental Modelling \& Software 22: 1509-1518. DOI: 10.1016/ j.envsoft.2006.10.004.

Lenhart T, Eckhardt K, Fohrer N, Frede H. 2002. Comparison of two different approaches of sensitivity analysis. Physics and Chemistry of the Earth, Parts $A / B / C$ 27: 645-654. DOI: 10.1016/S1474-7065(02)00049-9.

McCuen R. 1973. The role of sensitivity analysis in hydrologic modeling. Journal of Hydrology 18: 37-53. DOI: 10.1016/ 0022-1694(73)90024-3.

Moore RJ. 1985. The probability-distributed principle and runoff production at point and basin scales. Hydrological Sciences Journal 30: 273-297. DOI: 10.1080/02626668509490989.

Moore RJ. 2007. The PDM rainfall-runoff model. Hydrology and Earth System Sciences 11: 483-499. DOI: 10.5194/hess-11483-2007.

Morris M. 1991. Factorial sampling plans for preliminary computational experiments. Technometrics 33: 161-174. DOI: 10.1080/00401706.1991.10484804.

Nash JE, Sutcliffe JV. 1970. River flow forecasting through conceptual models part I - A discussion of principles. Journal of Hydrology 10: 282-290. DOI: 10.1016/0022-1694(70) 90255-6.

Saltelli A, Tarantola S, Campolongo F, Ratto M. 2004. Sensitivity analysis in practice: A guide to assessing scientific models. John Wiley \& Sons. DOI: 10.1002/0470870958.

Tang Y, Reed P, Wagener T, Van Werkhoven K. 2007. Comparing sensitivity analysis methods to advance lumped watershed model identification and evaluation. Hydrology and Earth System Sciences 11: 793-817. DOI: 10.5194/hess-11-7932007.

Vemuri V, Dracup J, Erdmann R, Vemuri N. 1969. Sensitivity analysis method of system identification and its potential in hydrologic research. Water Resources Research 5: 341-349. DOI: 10.1029/WR005i002p00341.

Zhao RJ. 1992. The Xinanjiang model applied in China. Journal of Hydrology 135: 371-381. DOI: 10.1016/0022-1694(92) 90096-E. 